\title{
On the Eruption of Prominences and Disappearance of Quiescent Filaments
}

\author{
S.S. Gupta \\ Indian Institute of Astrophysics, Kodaikanal - 624103, India \\ Jagdev Singh \\ Indian Institute of Astrophysics, Bangalore - 560034, India
}

\begin{abstract}
The analysis of an eruptive prominence and disappearing quiescent filaments indicates that the prominence height on the limb increases after the filament develops a twist as seen in the $\mathrm{H} \alpha$ on the disc. The increase in prominence height, large change in the shear angle, and the appearance of filament as a U-shaped structure before the disappearance of the filament or prominence eruption can be explained by assuming that the middle portion of the filament rises above the solar surface with its edges anchored in the chromosphere. Further, the loop-like structure of the filament splits into two parts 2-3 days prior to its disappearance and the gap between the two increases with time. The increasing gap and simultaneous disappearance of both parts of the filament suggests that heating of the top of the loop-like structure begins about 2 days before its disappearance. The cause for this portion of the filament not being visible in $\mathrm{H} \alpha$ may be that it is heated to a high temperature.
\end{abstract}

\section{Introduction}

Detailed observations of quiescent prominences over a long period can provide a clue to conditions for the onset of the eruptive instability, as well as its development (Vrsnak $1990 \mathrm{a}, \mathrm{b}$ ). To understand the mechanism and role of shear before the disappearance of quiescent filaments, we have investigated an eruptive prominence and two disappearing quiescent filaments, in the period December 1949 to April 1950, with a new technique for measuring the shear and orientation of the filament.

\section{Observational Data and Measuring Technique}

Data has been taken from the solar obsevational archives of Kodaikanal observatory, consisting of photoheliograms (image diameter of $200 \mathrm{~mm}$ ), spectroheliograms in $\mathrm{H} \alpha, \mathrm{Ca}+\mathrm{K}$ and prominences (image diameter of $60 \mathrm{~mm}$ ). For the three cases taken in this study, the positions of sunspots and sketches of the $\mathrm{H} \alpha$ filaments were marked on a $200 \mathrm{~mm}$ diameter sunchart. The coordinates of the different portions of the filament were measured from the sunchart and replotted on a linear graph to avoid the error in measurement due to foreshort- 


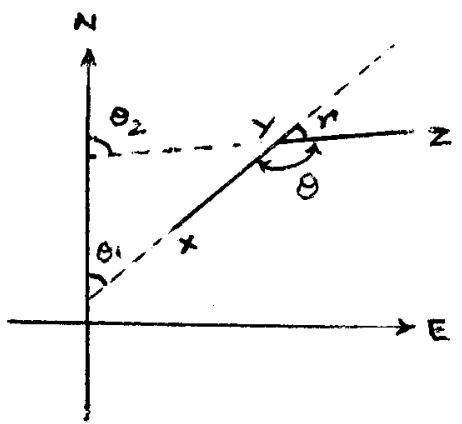

Figure 1. Technique used to measure the shear angle in $\mathrm{H} \alpha$ filaments.

ening. To detect changes in the filament orientation with time, the angle made by the filament or its each portion of the filament with the rotation axis of the sun was measured from the linear graph from the north towards the east. The total uncertainty in the measurement of angles due to pole marking, alignment of different images and sketching of filament is $\pm 2^{\circ}$ near the centre of the image and $\pm 4^{\circ}$ near the limb.

Consider a non-linear filament XYZ (Figure 1). The filament can be represented by two straight lines $X Y$ and $Y Z . \Theta_{1}$ and $\Theta_{2}$ are the angles made by these two portions with the rotation axis of the solar image, respectively. The angle between these two portions of filaments, $\Theta$, is given by the relation $\Theta=\Theta_{1}-\Theta_{2}+180^{\circ}$. The shear angle $\gamma=180^{\circ}-\Theta$, is defined as the amount by which the filament differs from a straight line. In the following we discuss the development of each filament in detail.

\section{Eruptive Prominence of February 3, 1950}

The prominence, mean location at $24^{\circ} \mathrm{SW}$, before it erupted at the west limb on February 3, was first visible as a filament on December 27, 1949. During its second transit on January 25, a kink at location 'A' (Figure 2) developed in the filament. The shear angle gradually increased until January 31 and then rapidly on February 1 and 2. Another kink developed at point ' $B$ ' on January 28 (Figure 2) and the shear angle at this point also increased. At the time of the first observation at 02:29:17 UT on February 3, this prominence was already in the eruptive phase and lasted for more than six hours. The eruptive phase ended with a part of the material falling back at a distant location in the vicinity of sunspot group at $10^{\circ} \mathrm{N}$.

\section{Disappearing Filament of February 1, 1950}

This quiescent filament survived less than one solar rotation. The filament located near the center of the solar disc at $10^{\circ} \mathrm{N}$ and $1^{\circ} \mathrm{W}$ disappeared between 08:36 UT on January 31 and 02:37 UT on February 1, 1950. It was first observed on January 25, 1950, as a prominence having a height of $55 \operatorname{arcsec}$ at a mean position of $10^{\circ} \mathrm{NE}$. The filament appeared sheared and broken at point ' $\mathrm{C}$ ' on January 28 (Figure 3 ). The shear angle remained $48^{\circ} \pm 6^{\circ}$ with minor fluctuations 

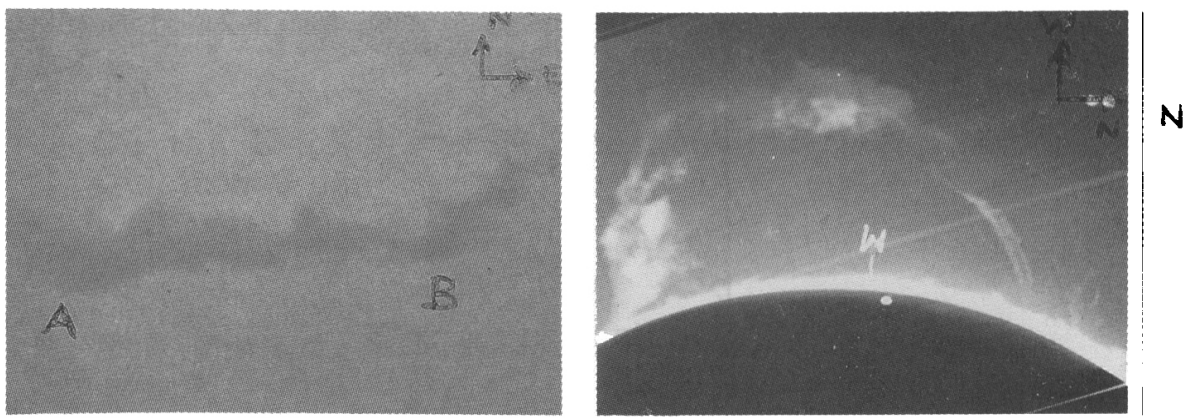

Figure 2. Left, $\mathrm{H} \alpha$ filament on January 30,1950 at 02:23:46 UT and, right, eruptive phase on the limb on February 3, 1950 at 04:16:00 UT.

until 02:56 UT on January 31. The gap between these two portions of the filament increased to about $5^{\circ}$ on January 31 . At 08:36 UT the shear angle became very large, $81^{\circ}$, and the filament very faint before disappearing.
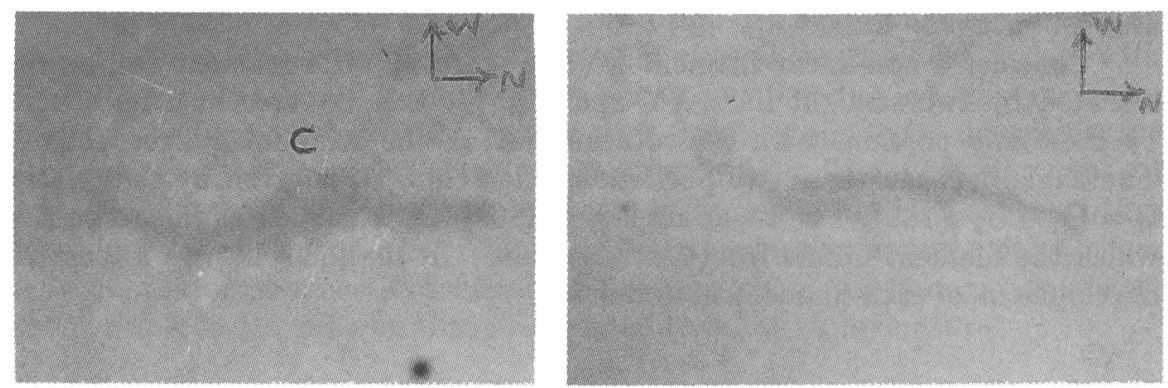

Figure 3. Left, $\mathrm{H} \alpha$ filament on January 29, 1950 at 02:19:08 UT and, right, on January 31,1950 at 02:56:08 UT.

\section{Disappearing Filament of April 15, 1950}

This quiescent filament lived more than 5 solar rotations. An inverted U-shaped $\mathrm{H} \alpha$ filament located at $23^{\circ}-43^{\circ} \mathrm{N}, 06^{\circ}-43^{\circ} \mathrm{E}$ on April 14 at $02: 45 \mathrm{UT}$ disappeared before 02:36 UT on April 15, 1950. The filament was first seen as a prominence at $30^{\circ} \mathrm{NE}$, on December 15, 1949. A small kink developed at point ' $D$ ' on January 16. The shear angle at this kink remained the same until February 19,1950 with small variations of the order of $\pm 7^{\circ}$. Correspondingly, it showed a small increase in height on the west and east limbs. A small portion on the west side of this filament disappeared on January 21 . The kink at point ' $\mathrm{D}$ ' developed from March 14 to 18, 1950 (Figure 4) and the shear angle during this period of 5 days was $42^{\circ} \pm 2^{\circ}$. Correspondingly, the prominence showed increase in height at the limbs.

In its fifth transit, the filament seen near the east limb on April 12 was highly sheared with a gap of about $5^{\circ}$. The filament took the shape of an inverted ' $U$ ' with the gap increasing to $12^{\circ}$ on April $13,17^{\circ}$ on April 14, (Figure 4) and the 

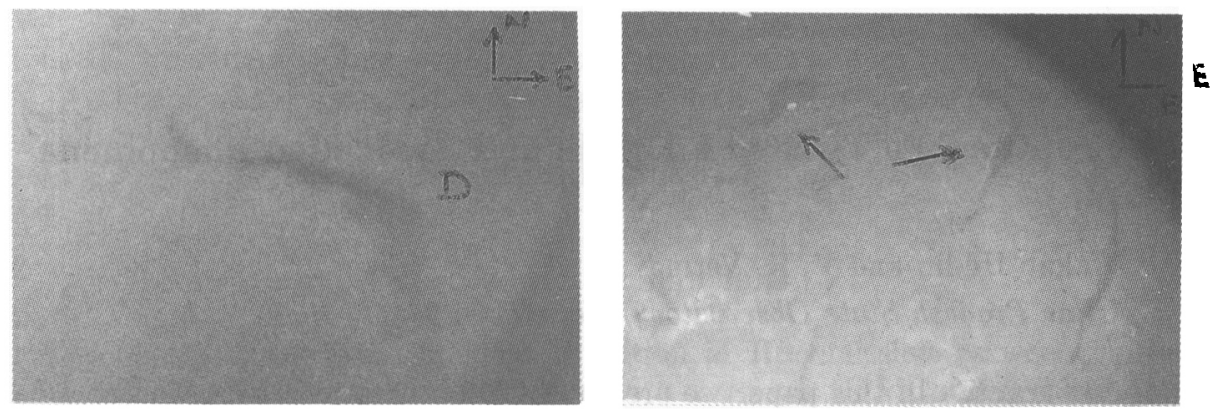

Figure 4. Left, $\mathrm{H} \alpha$ filament on March 12, 1950 at 08:13:36 UT and, right, on April 14, 1950 at 02:40:10 UT.

shear angle became $74^{\circ}$ and $103^{\circ}$, respectively. Between the afternoon of April 14 and morning of April 15 both portions of this filament disappeared.

\section{Discussion}

In all the three cases, the shear angles became very large one or two days before the disappearance of the filament. A change in the orientation of the filament or development of a kink causing shear in the filament can be due to the rise of the middle part of the filament above the solar surface. The possibility of this part of the filament rising above the solar surface, due to heating or other instability, and appearing as an inverted U-shaped structure due to projection and line of sight effects seems to be real. Thus, it appears that the measured shear in the filament is due to rise of the part of the filament above the solar surface and the ends of the filament anchored in the chromosphere.

We have seen that the $\mathrm{H} \alpha$ filament split into two portions 2-3 days prior to its disappearance and the gap between the two parts increased with time. Also, both portions of the filament disappeared at the same time and the middle part of the filament rose into the corona far above the solar surface before its disappearance. Rust (1984) and Tang (1986) reported that a filament disappearance may be caused by heating or by dynamic processes. Considering these facts, one may assume that the top of the filament gets heated and thus is not seen in $\mathrm{H} \alpha$ pictures. Simultaneous observations with good photometric accuracy in different temperature-sensitive lines will yield clues to understanding the circumstances leading to the eruption of quiescent prominences and filament disappearances.

\section{References}

Rust, D.M. 1984, Solar Phys., 93, 75

Tang, F. 1986, Solar Phys., 105, 2

Vrsnak, B. 1990a, Solar Phys., 127, 129

Vrsnak, B. 1990b, Solar Phys., 129, 295 